\title{
Mavacamten - a new disease-specific option for pharmacological treatment of symptomatic patients with hypertrophic cardiomyopathy
}

\author{
Piotr Pysz ${ }^{1 *}$, Renata Rajtar-Salwa2*, Grzegorz Smolka', lacopo Olivotto³, Wojciech Wojakowski', \\ Paweł Petkow-Dimitrow ${ }^{2,4}$
}

\author{
'Division of Cardiology and Structural Heart Diseases, Medical University of Silesia, Katowice, Poland \\ 2Department of Cardiology and Cardiovascular Interventions, University Hospital, Kraków, Poland \\ ${ }^{3}$ Cardiomyopathy Unit, Azienda Ospedaliera Universitaria Careggi, Florence, Italy \\ ${ }^{4} 2^{\text {nd }}$ Department of Cardiology, Jagiellonian University Medical College, Kraków, Poland \\ *Both authors equally contributed to the study
}

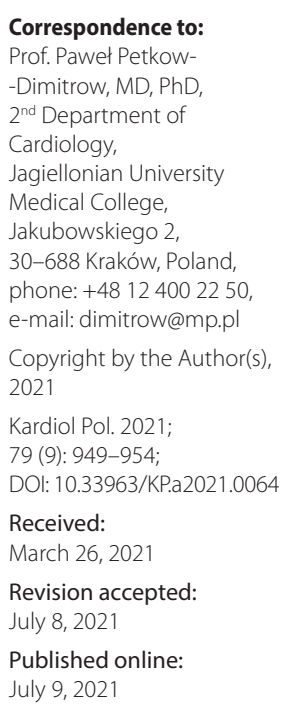

Correspondence to: Prof. Paweł Petkow-Dimitrow, MD, PhD, $2^{\text {nd }}$ Department of Cardiology,

Jagiellonian University Medical College, Jakubowskiego 2, 30-688 Kraków, Poland phone: +48 1240022 50, e-mail: dimitrow@mp.pl

Copyright by the Author(s), 2021

Kardiol Pol. 2021; 79 (9): 949-954;

DOI: 10.33963/KP.a2021.0064

Received:

March 26, 2021

Revision accepted: July 8, 2021

Published online: July 9, 2021

\begin{abstract}
A B S TR A C T
Current pharmacotherapy for hypertrophic cardiomyopathy (HCM) is not disease-specific and has suboptimal efficacy, often necessitating interventional treatment. EXPLORER-HCM was a phase 3, randomized, double-blind, placebo-controlled, multicenter clinical trial investigating the effects of mavacamten, a first-in-class selective cardiac myosin inhibitor, in patients with $\mathrm{HCM}$, left ventricular outflow tract obstruction (LVOTO) and New York Heart Association (NYHA) class II or III symptoms. The primary endpoint was defined as either $\mathrm{a} \geq 1.5 \mathrm{ml} / \mathrm{kg} / \mathrm{min}$ increase in peak oxygen consumption $\left(\mathrm{pVO}_{2}\right)$ and $\geq 1 \mathrm{NYHA}$ class reduction or $\mathrm{a} \geq 3.0 \mathrm{ml} / \mathrm{kg} / \mathrm{min} \mathrm{pVO}_{2}$ increase without NYHA class worsening. Secondary endpoints evaluated changes in post-exercise LVOT gradient, $\mathrm{pVO}_{2}$, NYHA class, Kansas City Cardiomyopathy Questionnaire-Clinical Summary Score (KCCQ-CSS), and Hypertrophic Cardiomyopathy Symptom Questionnaire Shortness-of-Breath subscore (HCMSQ-SoB). A total of 251 patients were randomized to receiving mavacamten or placebo. The primary endpoint and all secondary endpoints were met significantly more frequently in the mavacamten arm versus placebo. The safety profile of mavacamten was similar to that of placebo. In conclusion, disease-specific treatment with mavacamten in patients with obstructive HCM led to reduced LVOTO and improvement in both objective functional parameters and patient-related health status.
\end{abstract}

Key words: hypertrophic cardiomyopathy, left ventricular outflow obstruction, mavacamten

Kardiol Pol 2021; 79, 9: 949-954

\section{INTRODUCTION}

Hypertrophic cardiomyopathy (HCM) is the most common genetic cardiomyopathy, with a 1:500 prevalence in the general population [1]. The diagnosis of HCM is based on demonstrating significant thickening of at least one myocardial segment of the left ventricle. Regardless of the imaging technique (echocardiography, magnetic resonance, computed tomography), a cut-off value of $15 \mathrm{~mm}$ in diastole has been adopted. Additionally, in first-degree relatives of patients with confirmed $\mathrm{HCM}$, a wall thickness of $\geq 13 \mathrm{~mm}$ is deemed sufficient for diagnosis. The core pathomechanism of HCM is represented by the enhanced affinity between actin and myosin filaments leading to hypercontractility, left ventricular diastolic dysfunction, and in about $70 \%$ of patients, dynamic left ventricular outflow tract obstruction (LVOTO). So far, roughly 1500 mutations in about 11 genes encoding different proteins of the sarcomere were identified. Most frequently (>70\%), genes encoding the beta-myosin heavy chain-7 and myosin-binding C3 proteins are affected. Recent investigations in understudied populations offer local and global verification of the genetic mutation underlying $\operatorname{HCM}[2,3]$. The key features of HCM are unexplained wall thickening, abnormal relaxation with increased filling pressures, myocardial fibrosis. Clinical manifestations are heart failure (HF), atrial fibrillation (AF), ventricular arrhythmia, potentially leading to sudden cardiac death, syncope, and angina. HCM can have a nonobstructive (no-HCM) or more frequent obstructive (oHCM) form. Dynamic LVOTO related to systolic anterior motion (SAM) of the mitral valve can be present 
at rest or detected after proper physiological provocative test, i.e., exercise, especially in the upright position [4-7] in up to $70 \%$ cases (sum of resting obstructive and provoked obstructive gradients) [8, 9]. Medical treatment of the HCM with severe LVOTO (gradient of $\geq 50 \mathrm{~mm} \mathrm{Hg}$ ) has been challenging, with many patients experiencing poor quality of life and often drug side effects. As the disease-specific treatment for HCM is still lacking, the current therapeutic regimens are based on empiric use of drugs such as beta-blockers (BB), verapamil, or disopyramide. The medical therapy is effective, however often associated with drug side effects, in particular in the case of disopyramide. Patients with symptoms refractory to medical therapy usually require septal reduction therapies (SRTs) which remains the most effective treatment provided it is performed in experienced high-volume centers.

\section{UNMET CLINICAL NEEDS IN OHCM WITH LVOTO}

Based on the 2014 European Society of Cardiology (ESC) guidelines, pharmacotherapy aiming to relieve the LVOTO and improve symptoms encompasses BB, non-dihydropyridine calcium channel blockers (CCB), and disopyramide (unavailable in Poland). Similar medical management is recommended in recent American Heart Association/American College of Cardiology guidelines [10-12]. Such therapies often lead to a reduction of LVOT gradient and improvement of symptoms. Still, they are often associated with clinically significant side effects, especially if combination therapy is used (BB or CCB + disopyramide). Also, the use of CCB in patients with HF and very high LVOT gradients is problematic due to their vasodilatory effects.

In the presence of LVOTO, BB became the first-line pharmacotherapy $[13,14]$ preferred over verapamil, reducing LVOT gradient via a negative inotropic effect. Direct comparison between propranolol and verapamil revealed that propranolol was more effective in gradient reduction, but only verapamil was able to significantly decrease New York Heart Association (NYHA) class [15]. Verapamil utility in oHCM with high resting gradients is limited by its vasodilating effects that can, infrequently, worsen gradient and symptoms $[16,17]$. As such, we tend to avoid it in patients with high gradients and severe HF symptoms [16].

In the European HCM center of excellence [13, 14] nadolol is the first choice drug, considering its good tolerability, favorable electrophysiological profile, potent effect on gradients, and adequate $24-\mathrm{h}$ coverage. Conversely, in the American HCM center of expertise [16] bisoprolol is the first-choice BB due to the highest beta-1 selectivity. However, at high doses of BB needed for obstruction, relief might be poorly tolerated (hypotension, bradycardia, decreased atrio-ventricular conduction, chronotropic incompetence, exacerbation of the chronic obstructive pulmonary disease, or erectile dysfunction). Verapamil, but not BB, improved coronary endothelial dysfunction [18]. Overall, the efficacy and side effect profile of the current pharmacotherapy are not optimal. Established SRTs involve surgical septal myectomy (Morrow's operation) and transcatheter alcohol septal ablation. The first one is recommended in younger, active patients with a thicker interventricular septum (IVS), higher LVOT gradient, and concurrent structural heart disease warranting, e.g., simultaneous mitral valve repair or replacement. Alternatively, the percutaneous approach is considered more suitable in the elderly, with IVS thickness below $18 \mathrm{~mm}$. Septal reduction therapies effectively reduce symptoms in the majority ( $>95 \%$ myectomy and $>75 \%$ alcohol septal ablation) of patients, however, they must be performed in experienced high-volume centers and are not widely available.

All of the above are not targeting the underlying pathophysiology of the disease. The contractile apparatus is a promising target for novel drug development; therefore, investigation for a new drug reducing LVOT gradient was crucial. In 2016 a small-molecule allosteric inhibitor of myosin was discovered (initially coded MYK-461), currently named mavacamten [19]. In contrast to non-specific inotropic negative drugs, mavacamten is a specific compound targeting the primary molecular defect of the cardiomyocyte function [20-22].

\section{MECHANISM OF ACTION}

From the pathophysiological point of view, both oHCM and noHCM are characterized by hypercontractile left ventricular myocardium despite hypertrophy status. The molecular underpinnings of hypercontractility relate to an energetically inefficient myosin. Active myosin is a composite of two myosin heads with intertwined tails. During the myosin force production cycle, there is an autoinhibited state, sometimes referred to as a super-relaxed state. With certain myosin mutations, in the HCM sarcomere, this inhibited state is shortened, leading to hyperactivation and excess ATP utilization [23-25]. Mavacamten, a first-inclass oral small molecule, targets this process directly as an allosteric modulator of cardiac $\beta$-myosin, causing reversible inhibition of actin-myosin cross-bridging (Figure 1). In detail, mavacamten stabilizes this inhibited state, effectively lengthening the time that myosin is inactive [23-25]. Mavacamten binds to myosin, stabilizes the super-relaxed conformation, and shifts the kinetics of the actin-activated phosphate release step of myosin activation, thus decreasing ATPase activity and essentially slowing interaction of myosin with actin. Although there are multiple myosin forms, Mavacamten is specific for $\beta$-myosin heavy chain, expressed in the myocardium. Mavacamten demonstrated low clearance, a high volume of distribution, long terminal elimination half-life, and high bioavailability after oral intake cross-species. Simple four-species allometric scaling led to predicted plasma clearance, the volume of distribution, and half-life of $0.51 \mathrm{ml} / \mathrm{min} / \mathrm{kg} ; 9.5 \mathrm{l} / \mathrm{kg}$, and 9 days, respectively, in humans [26]. Importantly this molecule is cardiac-selective and does not influence the function of skeletal muscles. 


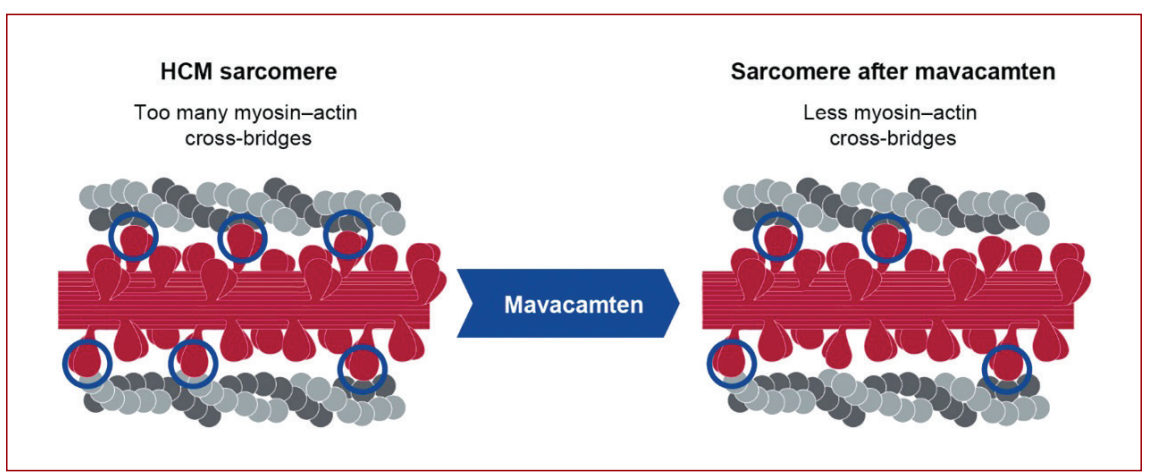

Figure 1. Mechanism of mavacamten action

\section{STUDIES IN ANIMAL MODELS}

Green et al. [19] demonstrated that chronic administration of MYK-461 during development suppresses the development of ventricular hypertrophy, cardiomyocyte disarray, myocardial fibrosis and attenuates hypertrophic and profibrotic gene expression in a mouse model of HCM. In the echocardiographic study in the feline genetic model of oHCM, treatment with mavacamten reduced contractility eliminated SAM, and relieved LVOTO [27]. The latest study assessed the effect of mavacamten on cardiac muscle contraction in two transgenic mouse lines expressing the human isoform of cardiac myosin regulatory light chain in their hearts [28]. Findings indicate that the drug reduces isometric tension and $\mathrm{Ca}^{2+}$-sensitivity of contraction via decreased strong cross-bridge binding.

\section{FIRST IN MAN STUDIES}

In the first study in patients, mavacamten was evaluated in a subgroup of oHCM in the open-label PIONEER-HCM (Pilot Study Evaluating drug in Subjects With Symptomatic Hypertrophic Cardiomyopathy and Left Ventricular Outflow Tract Obstruction) [29]. The PIONEER-HCM was a prospective, phase 2 study to evaluate the pharmacokinetics and pharmacodynamics of mavacamten. The inclusion criteria were resting LVOT gradient greater than 30 or provoked gradient greater than $50 \mathrm{~mm} \mathrm{Hg}$. The study included $21 \mathrm{pa}-$ tients. In subgroup A $(\mathrm{n}=11)$, higher-dose mavacamten resulted in a significant and rapid reduction in the degree of post-exercise LVOTO (average $\sim 90 \mathrm{~mm} \mathrm{Hg}$ decrease in post-exercise LVOT gradient). In this subgroup, there was also a substantial (17\%) improvement in exercise capacity (peak oxygen consumption $\left[\mathrm{VO}_{2}\right]+3.5 \mathrm{ml} / \mathrm{kg} / \mathrm{min}$ ). Subgroup $B(n=10)$ received lower doses of mavacamten. While improvement in LVOT obstruction (average $\sim 25 \mathrm{~mm}$ $\mathrm{Hg}$ decrease in post-exercise LVOT gradient) and exertional capacity (peak $\mathrm{VO}_{2}+1.7 \mathrm{ml} / \mathrm{kg} / \mathrm{min}$ ) were more modest, there was still significant symptom improvement. In both higher and lower doses of mavacantem, a substantial reduction in gradient was observed. Furthermore, eight participants had a decrease of LVOT gradient $<30 \mathrm{~mm} \mathrm{Hg}$. Apart from positive hemodynamic changes, a beneficial lowering of serum $\mathrm{N}$-terminal pro-brain natriuretic peptide
(NT-proBNP) was also observed (biomarker representing myocardial wall stress). Importantly, the left ventricular ejection fraction declined by $6 \%$ to $15 \%$, which corresponds with the expected mode of cellular action of the myosin inactivator. These positive findings set the stage for the recently presented EXPLORER-HCM (Clinical Study to Evaluate Mavacamten [MYK-461] in Adults With Symptomatic Obstructive Hypertrophic Cardiomyopathy) study.

\section{RANDOMIZED, MULTICENTER STUDIES}

EXPLORER-HCM is a multicenter, phase 3, randomized, double-blind, placebo-controlled trial to investigate the efficacy and safety of mavacamten in treating symptomatic 220 patients with oHCM. EXPLORER-HCM trial enrolled adult patients with an established diagnosis of HCM, maximal LVOT gradient exceeding $50 \mathrm{~mm} \mathrm{Hg}$ (at rest or provoked by Valsalva maneuver or exercise), preserved left ventricular ejection fraction (LVEF $\geq 55 \%$ ), and NYHA class II or III HF symptoms. Moreover, patients had to be capable of performing upright cardiopulmonary exercise testing. Main exclusion criteria included a history of exercise-induced syncope or sustained ventricular tachyarrhythmia (VT) within 6 preceding months; QT interval corrected using Fridericia's formula $>500 \mathrm{~ms}$, AF present on screening electrocardiogram, and any AF without anticoagulation for at least 4 weeks or with insufficient rate control within 6 months before screening. Prior septal reduction therapy did not exclude a patient, provided it was performed more than 6 months before screening. Continuation of standard HCM pharmacotherapy with a BB or calcium channel blocker was allowed as long as the dosing remained unchanged for at least 2 weeks before the screening, and no modifications were expected throughout the study. The base dose of mavacamten was $5 \mathrm{mg}$ qd and was individually up-titrated to a maximum of $15 \mathrm{mg}$ at weeks 8 and 14 to achieve the target reduction of LVOT gradient $<30 \mathrm{~mm} \mathrm{Hg}$ and mavacamten plasma concentration between $350 \mathrm{ng} / \mathrm{ml}$ and $700 \mathrm{ng} / \mathrm{ml}$. The primary composite endpoint was defined as an increase of $\mathrm{pVO}_{2}$ by at least $1.5 \mathrm{ml} / \mathrm{kg} / \mathrm{min}$ accompanied by at least one NYHA class reduction or an increase of $\mathrm{pVO}_{2}$ by at least $3.0 \mathrm{ml} / \mathrm{kg} / \mathrm{min}$ with no worsening of NYHA class. Among secondary endpoints, changes in post-exer- 
cise LVOT gradient, $\mathrm{pVO}_{2}$, percentage of patients with at least one NYHA class improvement, and cardiomyopathy questionnaires (KCCQ-CSS, HCMSQ-SoB) were evaluated.

Eligible adults with oHCM and NYHA were randomized $1: 1$ to receive once-daily, oral mavacamten, or matching placebo for 30 weeks [30]. 98\% of patients enrolled completed the study. Of the $2 \%$ who dropped out, none was lost due to reduced LVEF or symptoms of HF. Overall rates of adverse events, serious adverse events, and cardiac adverse events, including AF, were comparable for patients treated with mavacamten and placebo. Safety and tolerability of the agent were similar to placebo. One patient died by sudden death in the placebo group.

\section{Main results}

The primary endpoint was achieved by 45 (37\%) patients in the mavacamten group and $22(17 \%)$ in the placebo group (+19.4\%; 95\% Cl, 8.7-30.1; $P=0.0005)$. Additionally, in the mavacamten group, an increase of $\mathrm{pVO}_{2}$ by at least $3.0 \mathrm{ml} / \mathrm{kg} / \mathrm{min}$ together with at least one NYHA class improvement was noted in 45 (37\%) patients, while the same effect in the placebo group was found in 10 patients (difference $+12.5 \%$; 95\% Cl, 4.0-21.0).

Secondary endpoints also pointed at the improvement associated with mavacamten administration. The decrease in post-exercise LVOT gradient in the treated group was greater by $35.6 \mathrm{~mm} \mathrm{Hg}(95 \% \mathrm{Cl},-43.2$ to $-28.1 ; P<0.0001)$ with a mean reduction from $86 \mathrm{~mm} \mathrm{Hg}$ to $38 \mathrm{~mm} \mathrm{Hg}$ vs $84 \mathrm{~mm} \mathrm{Hg}$ to $73 \mathrm{~mm} \mathrm{Hg}$. The $\mathrm{pVO}_{2}$ increase with mavacamten was $1.4 \mathrm{ml} / \mathrm{kg} / \mathrm{min}$ greater than with placebo $(95 \% \mathrm{Cl}$, $0.6-2.1 ; P=0.0006) .80$ (65\%) patients treated with mavacamten noted an improvement by at least one NYHA class vs 40 (31\%) patients receiving placebo (difference 33.8\%; 95\% $\mathrm{Cl}, 22.2-45.4 ; P<0.0001)$. Furthermore, in the mavacamten group 61 (50\%) patients achieved NYHA class I, whereas, in the placebo group, there were 27 (21\%) of them. Correspondingly, also patient-reported outcomes as assessed by KCCQ-CCS (positive change better) and HCMSQ-SoB (negative change better) favored treatment with mavacamten (KCCQ-CCS +9.1; 95\% Cl, 5.5-12.7; HCMSQ-SoB -1.8;-2.4 to $-1.2 ; P<0.0001$ for both). Complete response was defined as a reduction of LVOT gradient $<30 \mathrm{~mm} \mathrm{Hg}$ together with achieving NYHA class I. Such a result was noted in 32 (27\%) patients treated with mavacamten, and only $1(1 \%)$ receiving placebo (+26.6\%; 95\% Cl, 18.3-34.8). Reduction of LVOT gradient below the standard cut-off value for interventional treatment that is $<50 \mathrm{~mm} \mathrm{Hg}$ was achieved by $53 \%$ more often in the mavacamten group (74.3\% vs $20.8 \%$ [difference $53.5 \%$; $95 \% \mathrm{Cl}, 42.0$ to $65.0, \mathrm{P}<0.001)$ ].

Notably, a significant number of patients on placebo had improvement in the primary endpoint and NYHA class. This highlights the need for proper double-blinding in such studies, which evaluate the physical performance and HF symptoms. On the other hand, the reduction of the NT-proBNP observed in the mavacamten group supported the conclusion that the differences between the groups were truly significant. Also, the reduction of the LVOT gradient was by $35 \%$ greater on mavacamten than on placebo and all of the secondary endpoints were consistently showing the superiority of the drug over placebo. Another important issue is the variability of the LVOT gradients. The variability tends to be higher for resting gradients and less for gradients provoked by exercise. The post-exercise gradient was the secondary endpoint of the trial, which makes the variability less relevant.

The reduction of cardiac biomarkers concentrations was similarly rapid and sustained, parallel to the hemodynamic changes noted. At week 30 compared with baseline, the drop in NT-proBNP after mavacamten treatment was $80 \%$ greater than for placebo, and the decrease in hs-cTnl was $41 \%$ greater for mavacamten than for placebo.

\section{NONOBSTRUCTIVE HCM}

Another direction of investigation (MAVERICK-HCM study) was designed to evaluate the dosing of mavacamten in HCM without LVOTO since the drug has extended pharmacokinetic properties [31]. Fifty nine patients were enrolled and observed for over 16 weeks. Patients were subdivided into 3 subgroups ( $n=19$ allocated to serum drug concentration of $\sim 200 \mathrm{ng} / \mathrm{ml}, \mathrm{n}=21$ allocated to $\sim 500 \mathrm{ng} / \mathrm{ml}$, and $\mathrm{n}=19$ to placebo), followed by an 8-week washout period. The MAVERICK-HCM study fulfilled its primary objective concerning safety and tolerability. The drug was well tolerated, with no differences in reported serious adverse events (SAE) between treatment groups (10\% on mavacamten and $21 \%$ on placebo). A common SAE was AF ( $5 \%$ in both groups), and the majority of other side effects (>70\%) were not clinically relevant. The main secondary outcome was a reduction in plasma biomarkers. In the pooled mavacamten groups, the NT-proBNP decreased by $53 \%$ vs $1 \%$ in the placebo group $(-435 \mathrm{pg} / \mathrm{ml} \mathrm{vs}-6 \mathrm{pg} / \mathrm{ml}$; $P=0.0005)$. Similarly, the cardiac troponin I also decreased by $34 \%$ in the pooled-mavacamten group compared to a $4 \%$ increase in the placebo group $(P=0.009)$. The investigators evaluated exploratory functional endpoints, including peak $\mathrm{VO}_{2}$ or NYHA functional class. Among a high-risk subgroup with elevated myocardial injury biomarkers (cardiac troponin I $>99^{\text {th }}$ percentile) or elevated diastolic filling pressures (average E/e' $>14$ on Doppler-echocardiogram), one-third of mavacamten treated patients met the composite functional endpoint defined as achieving: (1) an improvement of at least $1.5 \mathrm{ml} / \mathrm{kg} / \mathrm{min}$ in peak $\mathrm{VO}_{2}$ with a reduction of NYHA functional class; or (2) an improvement of $3.0 \mathrm{ml} / \mathrm{kg} / \mathrm{min}$ or more in peak $\mathrm{VO}_{2}$ with no worsening in NYHA functional class - compared with none in the placebo group $(P=0.03)$. The effect on the plasma level of both biomarkers is particularly beneficial given recent evidence demonstrating the unfavorable role of persistently high levels of these biomarkers in $\operatorname{HCM}[32,33]$. 


\section{IMAGING STUDIES}

The recent cardiac magnetic resonance substudy of EXPLORER [34] is the first to show the favorable influence of mavacamten on cardiac remodeling in HCM. Mavacamten was associated with a significant reduction in the total intracellular myocardial mass index as well as left ventricular (LV) mass index, maximum LV wall thickness, and left atrial volume index. Importantly, no changes in fibrosis level were observed over 30 weeks. Furthermore, the contractile function was maintained in the normal range. Reduction in hypertrophy and left atrial volume were demonstrated parallelly with a decrease in plasma levels of biomarkers of myocardial stress and injury.

\section{ONGOING STUDIES}

The findings of EXPLORER-HCM and MAVERICK-HCM studies are further confirmed in two ongoing long-term extension studies - A Long-term Safety Extension Study of Mavacamten (MYK461) in Adults with Hypertrophic Cardiomyopathy Who Have Completed the MAVERICK-HCM (MYK-461-006) or EXPLORER-HCM (MYK-461-005) Trials (MAVA-LTE).

The main goals of LTE are: (1) assessment of the long-term safety and tolerability of mavacamten in participants with HCM who completed the prior studies; (2) collection of data on the long-term outcomes in patients with symptomatic noHCM and oHCM; and (3) evaluation of the echo-guided mavacamten titration algorithm in symptomatic oHCM participants. The active treatment in both studies is 2 years, and all patients receive the drug.

VALOR-HCM (Evaluation of Mavacamten in Adults with Symptomatic Obstructive Hypertrophic Cardiomyopathy who are Eligible for Septal Reduction Therapy) study will verify the hypothesis that in patients $(n=100)$ with symptomatic oHCM the course of mavacamten over 16 week will reduce the need for SRTs [35]. Until then SRTs remain the most effective treatment for drug-refractory oHCM [36].

\section{CLINICAL PERSPECTIVE}

Mavacamten seems to be a useful drug for the full spectrum of HCM presentations (obstructive and nonobstructive). Beneficial effects include LVOT gradient reduction, an increase of exercise tolerance, and a decreased level of biomarkers (reduction of wall stress and ischemia injury). Also, clinical status and quality of life tend to follow these hemodynamic changes. Mavacamten is the first in class drug targeting the key underlying molecular mechanisms of cardiomyocyte hypercontractility in HCM.

\section{Article information}

Conflicts of interest: IO: grants, research funding: Myokardia, Cytokinetics, Amicus, Shire Takeda, Sanofi Genzyme, Bayer, Menarini International, Boston Scientific. Advisory board/speakers'fees: Myokardia, Cytokinetics, Amicus, Shire Takeda, Sanofi Genzyme. WW: Advisory board Myokardia. Other authors declare no conflicrtof interest.
Open access: This article is available in open access under Creative Common Attribution-Non-Commercial-No Derivatives 4.0 International (CC BY-NC-ND 4.0) license, allowing to download articles and share them with others as long as they credit the authors and the publisher, but without permission to change them in any way or use them commercially. For commercial use, please contact the journal office at kardiologiapolska@ptkardio.pl.

How to cite: Pysz P, Rajtar-Salwa R, Smolka G, et al. Mavacamten - a new disease-specific option for pharmacological treatment of symptomatic patients with hypertrophic cardiomyopathy. Kardiol Pol. 2021; 79(9): 949-954, doi: 10.33963/KP.a2021.0064.

\section{REFERENCES}

1. Maron BJ. Clinical course and management of hypertrophic cardiomyopathy. N Engl J Med. 2018;379(7):655-668, doi: 10.1056/NEJMra1710575, indexed in Pubmed: 30110588

2. Lipari M, Wypasek E, Karpinski M, et al. Identification of a variant hotspot in MYBPC3 and of a novel CSRP3 autosomal recessive alteration in a cohort of Polish cases affected by hypertrophic cardiomyopathy. Pol Arch Intern Med. 2020; 130(2): 89-99, doi: 10.20452/pamw.15130, indexed in Pubmed: 31919335.

3. Walsh R, Bezzina CR. Research in understudied populations offers local and global insights into the genetics of hypertrophic cardiomyopathy. Pol Arch Intern Med. 2020; 130(2): 76-78, doi: 10.20452/pamw.15214, indexed in Pubmed: 32105245.

4. Dimitrow PP, Rajtar-Salwa R. Obstructive form of hypertrophic cardiomyopathy-left ventricular outflow tract gradient: novel methods of provocation, monitoring of biomarkers, and recent advances in the treatment. Biomed Res Int. 2016; 2016: 1575130, doi: 10.1155/2016/1575130, indexed in Pubmed: 27247935.

5. Smarż K, Zaborska B, Pilichowska-Paszkiet E, et al. Significant left ventricular outflow tract obstruction observed during postexercise verticalization in a symptomatic patient with hypertrophic cardiomyopathy. Kardiol Pol. 2019; 77(6): 655-656, doi: 10.33963/KP.14860, indexed in Pubmed: 31169812.

6. Dimitrow P, Rajtar-Salwa R, Tokarek T. Exercise stress test methodology and safety in hypertrophic cardiomyopathy. Eur J Prev Cardiol. 2020; 27(17): 1819-1820, doi: 10.1177/2047487319901059, indexed in Pubmed: 32013606.

7. Reant $P$, Dufour M, Peyrou J, et al. Upright treadmill vs. semi-supine bicycle exercise echocardiography to provoke obstruction in symptomatic hypertrophic cardiomyopathy: a pilot study. Eur Heart J Cardiovasc Imaging. 2018; 19(1): 31-38, doi: 10.1093/ehjci/jew313, indexed in Pubmed: 28329285

8. Maron MS, Olivotto I, Zenovich AG, et al. Hypertrophic cardiomyopathy is predominantly a disease of left ventricular outflow tract obstruction. Circulation. 2006; 114(21): 2232-2239, doi: 10.1161/CIRCULATIONAHA.106.644682, indexed in Pubmed: 17088454.

9. Dimitrow PP, Cheng TO. Standing position alone or in combination with exercise as a stress test to provoke left ventricular outflow tract gradient in hypertrophic cardiomyopathy and other conditions. Int J Cardiol. 2010; 143(3): 219-222, doi: 10.1016/j.jijcard.2010.04.026, indexed in Pubmed: 20442001.

10. Elliott PM, Anastasakis A, Borger MA, et al. Authors/Task Force members. 2014 ESC Guidelines on diagnosis and management of hypertrophic cardiomyopathy: the Task Force for the Diagnosis and Management of Hypertrophic Cardiomyopathy of the European Society of Cardiology (ESC). Eur Heart J. 2014; 35(39):2733-2779, doi: 10.1093/eurheartj/ehu284, indexed in Pubmed: 25173338.

11. Sherrid MV, Barac I, McKenna WJ, et al. Multicenter study of the efficacy and safety of disopyramide in obstructive hypertrophic cardiomyopathy. J Am Coll Cardiol. 2005; 45(8): 1251-1258, doi: 10.1016/j.jacc.2005.01.012, indexed in Pubmed: 15837258.

12. Sherrid MV, Shetty A, Winson G, et al. Treatment of obstructive hypertrophic cardiomyopathy symptoms and gradient resistant to first-line therapy with $\beta$-blockade or verapamil. Circ Heart Fail. 2013; 6(4): 694-702, doi: 10.1161/CIRCHEARTFAILURE.112.000122, indexed in Pubmed: 23704138. 
13. Ammirati E, Contri R, Coppini R, et al. Pharmacological treatment of hypertrophic cardiomyopathy: current practice and novel perspectives. Eur J Heart Fail. 2016; 18(9): 1106-1118, doi: 10.1002/ejhf.541, indexed in Pubmed: 27109894.

14. Nistri $\mathrm{S}$, Olivotto I, Maron MS, et al. $\beta$ Blockers for prevention of exercise-induced left ventricular outflow tract obstruction in patients with hypertrophic cardiomyopathy. Am J Cardiol. 2012; 110(5): 715-719, doi: 10.1016/j.amjcard.2012.04.051, indexed in Pubmed: 22633205.

15. Kober G, Hopf R, Biamino G, et al. Long-term treatment of hypertrophic cardiomyopathy with verapamil or propranolol in matched pairs of patients: results of a multicenter study.Z Kardiol. 1987; 76(Suppl 3):113-118, indexed in Pubmed: 3324526.

16. Sherrid MV. Drug therapy for hypertrophic cardiomypathy: physiology and practice. Curr Cardiol Rev. 2016; 12(1): 52-65, doi: 10.2174/1573403 x1201160126125403, indexed in Pubmed: 26818487.

17. Epstein SE, Rosing DR. Verapamil: its potential for causing serious complications in patients with hypertrophic cardiomyopathy. Circulation. 1981; 64(3):437-441, doi: 10.1161/01.cir.64.3.437, indexed in Pubmed: 7196300.

18. Dimitrow PP, Krzanowski M, Nizankowski $R$, et al. Comparison of the effect of verapamil and propranolol on response of coronary vasomotion to cold pressor test in symptomatic patients with hypertrophic cardiomyopathy. Cardiovasc Drugs Ther. 2000; 14(6): 643-650, doi: 10.1023/a:1007871032421, indexed in Pubmed: 11300365.

19. Green EM, Wakimoto $H$, Anderson $R L$, et al. A small-molecule inhibitor of sarcomere contractility suppresses hypertrophic cardiomyopathy in mice. Science. 2016; 351(6273): 617-621, doi: 10.1126/science.aad3456, indexed in Pubmed: 26912705.

20. Tuohy CV, Kaul S, Song HK, et al. Hypertrophic cardiomyopathy: the future of treatment. Eur J Heart Fail. 2020; 22(2): 228-240, doi: 10.1002/ejhf.1715, indexed in Pubmed: 31919938.

21. Fumagalli $C$, De Gregorio MG, Zampieri M, et al. Targeted medical therapies for hypertrophic cardiomyopathy. Curr Cardiol Rep. 2020; 22(2): 10, doi: 10.1007/s11886-020-1258-x, indexed in Pubmed: 31993794.

22. Alsulami K, Marston S. Small molecules acting on myofilaments as treatments for heart and skeletal muscle diseases. Int J Mol Sci. 2020; 21(24): 9599, doi: 10.3390/ijms21249599, indexed in Pubmed: 33339418.

23. Kawas RF, Anderson RL, Ingle SRB, et al. A small-molecule modulator of cardiac myosin acts on multiple stages of the myosin chemomechanical cycle. J Biol Chem. 2017; 292(40): 16571-16577, doi: 10.1074/jbc. M117.776815, indexed in Pubmed: 28808052.

24. Rohde JA, Roopnarine O, Thomas DD, et al. Mavacamten stabilizes an autoinhibited state of two-headed cardiac myosin. Proc Natl Acad Sci USA. 2018; 115(32):E7486-E7494, doi: 10.1073/pnas.1720342115, indexed in Pubmed: 30018063.

25. Anderson RL, Trivedi DV, Sarkar SS, et al. Deciphering the super relaxed state of human $\beta$-cardiac myosin and the mode of action of mavacamten from myosin molecules to muscle fibers. Proc Natl Acad Sci U S A. 2018; 115(35): E8143-E8152, doi: 10.1073/pnas.1809540115, indexed in Pubmed: 30104387.
26. Grillo MP, Erve JCL, Dick R, et al. In vitro and in vivo pharmacokinetic characterization of mavacamten, a first-in-class small molecule allosteric modulator of beta cardiac myosin. Xenobiotica. 2019; 49(6): 718-733, doi: 10.1080/00498254.2018.1495856, indexed in Pubmed: 30044681.

27. Stern JA, Markova S, Ueda Y, et al. A small molecule inhibitor of sarcomere contractility acutely relieves left ventricular outflow tract obstruction in feline hypertrophic cardiomyopathy. PLoS One. 2016; 11(12): e0168407, doi: 10.1371/journal.pone.0168407, indexed in Pubmed: 27973580.

28. Awinda PO, Watanabe M, Bishaw Y, etal. Mavacamten decreases maximal force and Ca sensitivity in the N47K-myosin regulatory light chain mouse model of hypertrophic cardiomyopathy. Am J Physiol Heart Circ Physiol. 2021; 320(2): H881-H890, doi: 10.1152/ajpheart.00345.2020, indexed in Pubmed: 33337957.

29. Heitner SB, Jacoby D, Lester SJ, et al. Mavacamten treatment for obstructive hypertrophic cardiomyopathy: a clinical trial. Ann Intern Med. 2019; 170(11):741-748, doi: 10.7326/M18-3016, indexed in Pubmed: 31035291.

30. Olivotto I, Oreziak A, Barriales-Villa R, et al. EXPLORER-HCM study investigators. Mavacamten for treatment of symptomatic obstructive hypertrophic cardiomyopathy (EXPLORER-HCM): a randomised, double-blind, placebo-controlled, phase 3 trial. Lancet. 2020; 396(10253): 759-769, doi: 10.1016/S0140-6736(20)31792-X, indexed in Pubmed: 32871100.

31. Ho CY, Mealiffe ME, Bach RG, et al. Evaluation of mavacamten in symptomatic patients with nonobstructive hypertrophic cardiomyopathy. J Am Coll Cardiol. 2020; 75(21): 2649-2660, doi: 10.1016/j.jacc.2020.03.064, indexed in Pubmed: 32466879.

32. Rajtar-Salwa R, Hładij R, Dimitrow PP. Elevated level of troponin but not $\mathrm{N}$-terminal probrain natriuretic peptide is associated with increased risk of sudden cardiac death in hypertrophic cardiomyopathy calculated according to the ESC guidelines 2014. Dis Markers. 2017; 2017: 9417908, doi: 10.1155/2017/9417908, indexed in Pubmed: 29358842.

33. Rajtar-Salwa R, Gębka A, Dziewierz A, et al. Hypertrophic cardiomyopathy: the time-synchronized relationship between ischemia and left ventricular dysfunction assessed by highly sensitive troponin I and NT-proBNP. Dis Markers. 2019; 2019: 6487152, doi: 10.1155/2019/6487152, indexed in Pubmed: 31320942

34. Saberi S, Cardim N, Yamani M, et al. Mavacamten favorably impacts cardiac structure in obstructive hypertrophic cardiomyopathy: EXPLORER-HCM cardiac magnetic resonance substudy analysis. Circulation. 2021; 143(6): 606-608, doi: 10.1161/CIRCULATIONAHA.120.052359, indexed in Pubmed: 33190524.

35. Desai MY, Wolski K, Owens A, et al. Study design and rationale of VALOR-HCM: evaluation of mavacamten in adults with symptomatic obstructive hypertrophic cardiomyopathy who are eligible for septal reduction therapy. Am Heart J. 2021 -89. 3; 239: 80-89, doi: 10.1016/j. ahj.2021.05.007, indexed in Pubmed: 34038706.

36. Dorobantu L, Ticulescu R, Greavu M, et al. Current management and surgical advances in patients with hypertrophic obstructive cardiomyopathy. Kardiol Pol. 2019; 77(9):829-836, doi: 10.33963/KP.14965, indexed in Pubmed: 31495825. 